\title{
Dynamic aperture extrapolation in the presence of tune modulation
}

\author{
M. Giovannozzi, ${ }^{1, *}$ W. Scandale, ${ }^{2}$ and E. Todesco ${ }^{3}$ \\ ${ }^{1}$ INFN, Via Irnerio 46, 40126 Bologna, Italy \\ ${ }^{2}$ CERN, SL Division, CH 1211 Geneva, Switzerland \\ ${ }^{3}$ Dipartimento di Fisica, University of Bologna, Via Irnerio 46, 40126 Bologna, Italy
}

(Received 4 August 1997)

\begin{abstract}
In hadron colliders, such as the Large Hadron Collider (LHC) to be built at CERN, the long-term stability of the single-particle motion is mostly determined by the field-shape quality of the superconducting magnets. The mechanism of particle loss may be largely enhanced by modulation of betatron tunes, induced either by synchrobetatron coupling (via the residual uncorrected chromaticity), or by unavoidable power supply ripple. This harmful effect is first investigated in a simple dynamical system model, the Hénon map with modulated linear frequencies. Then a realistic accelerator model describing the injection optics of the LHC lattice is analyzed. Orbital data obtained with long-term tracking simulations $\left(10^{5}-10^{7}\right.$ turns $)$ are post-processed to obtain the dynamic aperture. It turns out that the dynamic aperture can be interpolated using a simple empirical formula, as it decays proportionally to a power of the inverse logarithm of the number of turns. Furthermore, the extrapolation of tracking data at $10^{5}$ turns gives reliable estimates of the dynamic aperture for $10^{7}$ turns, which represent the expected duration of the LHC injection plateau. [S1063-651X(98)00602-3]

PACS number(s): 41.85.- p, 29.27.- a, 03.20.+i
\end{abstract}

\section{INTRODUCTION}

In large hadron accelerators, such as the Large Hadron Collider (LHC) [1], two counter-rotating beams perform up to $10^{7}$ turns at the injection plateau, before energy ramping. During this time, the particle dynamics can be dominated by the unavoidable field-shape imperfections of the superconducting magnets. The nonlinear fields endanger the beam stability, since they introduce a tune dependence with amplitude and energy, and excite nonlinear resonances [2-5]. The resulting particle losses may also occur after many millions of turns (see, for instance, Refs. [6-10]).

To optimize the accelerator performances and to fix an upper bound to the unwanted multipolar errors, one has to evaluate the border of the region around the reference orbit that is stable for the required number of turns. Inside this domain, usually called the dynamic aperture, one can safely operate with the beam. The evaluation of the dynamic aperture is in general based on symplectic integration of complicated equations of motion using tracking codes. Notwithstanding the powerful farms of modern computers, for a realistic model of the LHC lattice one can hardly evaluate the beam stability for more than $10^{6}$ turns. Moreover, one has to investigate a large set of initial conditions for each machine configuration, and several configurations to optimize the lattice performances. Therefore, to design the LHC lattice for $10^{7}$ turns, methods alternative to brute-force element-by-element tracking should be worked out.

Long-term particle losses are drastically enhanced if the betatron tune is modulated by some external causes, such as the power supply ripple, or by synchrobetatron coupling, via the residual uncompensated chromaticity. This effect can be modelized by a set of nonlinear oscillators whose linear fre-

\footnotetext{
*Present address: PS Division, CERN, CH 1211 Geneva, Switzerland.
}

quencies are modulated. There exists a wide literature on this subject: analytical and numerical studies have been carried out on both simplified and more realistic models of accelerators; numerous experiments have been performed on several machines [10-13].

Mechanisms for modulational diffusion were analyzed in Refs. $[14,15]$; using perturbation theory one can work out analytical estimates of the diffusion coefficients in the modulational layer created by resonance overlapping. These tools have been used to analyze a simplified model (modulated FODO cell, i.e., a modulated Hénon mapping) to distinguish between different regimes due to the modulational spectra [16]. Rigorous estimates have also been obtained for the change in the adiabatic invariant when the phase space is slowly swept by a resonance [17]. Using these results, estimates of the diffusion coefficient in some phase space regions have been worked out $[18,19]$.

If the mechanism of stability loss can be described in terms of a Fokker-Planck equation, the diffusion coefficient has to depend strongly on the adiabatic invariants, and on the local structure of the resonances (see, for instance, Ref. [15]). This leads to severe difficulties, since in order to treat the complete problem one has to consider at least the underlying network of resonances in the four-dimensional (4D) phase space, while most of the references are restricted to the analysis of a 2D case (plus modulation). Approximations that assume a smooth dependence of the diffusion coefficient on the invariants [12] clearly neglect the presence of the wellknown phenomena due to the local structure of resonances [10]; on the other hand, the relevance of these local phenomena can be questionable. The possibility itself of describing the dynamics in presence of tune modulation through a diffusive process, i.e., with a Fokker-Planck equation and an action-dependent diffusion coefficient, has been considered questionable by some authors [20].

Other approaches to the analysis of long-term stability have been proposed. The Lyapunov exponent (originally de- 
veloped and applied in celestial mechanics [21-23]) is an indicator that can be extracted from tracking data: it should allow one to distinguish regular from chaotic motion using a limited number of turns $[8,9]$. Assuming that all chaotic particles will be lost, one obtains an estimate of the core of stable particles. Unfortunately, this assumption leads to severe underestimates in the case of tune modulation, where particle losses can be extremely slow, and therefore chaotic regions are stable for a sufficiently high number of turns [10]. One can also try to define approximated global invariants through numerical methods, and use the drift in the space of these pseudoinvariants to give long-term bounds $[24,25]$.

A pragmatic approach is based on the analysis of the survival times provided by computer simulations, plotted versus the initial amplitude (survival plots; see Refs. $[6,7,26]$ ). The main drawback is that these plots are very irregular, and therefore an extrapolation to larger times is very hard to be obtained (see, for instance, Refs. $[6,7,20,26])$. This is due to an insufficient analysis of the phase space, which is usually scanned along a line $I_{x}=I_{y}$ in the space of the linear invariants $\left(I_{x}, I_{y}\right)$; in a previous paper we showed that, by performing a scan of the phase space along several lines $I_{y}$ $=I_{x} \tan \alpha$, and taking an average over the angle $\alpha$ [27], the survival plots become much more regular.

For the purely $4 \mathrm{D}$ case (i.e., no tune modulation) the dynamic aperture turns out to approximately decay with the inverse of the logarithm of the number of turns [9]

$$
D(N)=A+\frac{B}{\log _{10} N} .
$$

This result can be interpreted through a simple dynamical model [28]: the phase space is divided into an inner region stable for infinite times, and an outer wide chaotic band where the escape rate agrees qualitatively with the Nekhoroshev estimate [29-31]. This approach turns out to be particularly useful since it provides quantitative estimates for large but finite number of turns. Some analyses of simplified 4D models [32,33] gave a first indication that the extrapolation of the inverse logarithm formula for infinite number of turns, i.e., the parameter $A$ in Eq. (1), agrees rather well with the prediction of the chaotic border obtained through the Lyapunov exponent.

In this paper we analyze the dynamics in the presence of tune modulation following a similar approach to the above cited 4D case. We make numerical simulations over both simplified lattices (the modulated 4D Hénon map, also analyzed in Refs. $[16,10])$ and realistic models of the LHC. Our purpose is to find a framework to interpret tracking data and to speed up, if possible, numerical simulations. Contrary to Ref. [16], we fix the spectrum of the modulational frequencies to the measured values for the super-proton-synchrotron (SPS) lattice [10]: we limit ourselves to finding the dependence of the dynamics on the amplitude of the modulation, for a given frequency spectra. Since we do not assume a diffusion process, we do not compute diffusion coefficients: the dynamical quantity that we evaluate is the dynamic aperture as defined in Ref. [27]. The averaging procedure used in this definition fails, in general, to detect the fine and irregular structure of resonance bands in phase space (see, for instance, Ref. [10]). The obtained dynamic aperture $D$ turns out to be a smooth function of the number of turns $N$, and is very well interpolated by the three-parameter formula

$$
D(N)=A+\frac{B}{\log _{10}^{\kappa} N} .
$$

For the 4D case the best fit of $\kappa$ is around 1.5, that is, the same value that can be rigorously proved through careful estimates of the remainder perturbative series [40]. The exponent decreases when the amplitude of the tune modulation becomes larger; this is natural, since larger modulational amplitudes imply more relevant long-term phenomena.

The above equation implies that the phase space is split into two parts: an inner part stable for infinite times, and an outer part where the chaotic motion is dominant. In the inner part the instabilities due to Arnold diffusion (or thin layer diffusion; see Ref. [15]) are negligible, since they occupy an irrelevant fraction of the phase space and, moreover, are extremely slow [41]. Using the terminology of Ref. [15], the outer part can be considered as a thick layer diffusion, where resonance overlapping has wiped out most of the invariant surfaces. It is not clear whether the decaying rate of the dynamic aperture empirically worked out by tracking data (2) fits with any diffusive process; the aim of this paper is to present a phenomenological framework to interpret the results of our numerical simulations, and to analyze the possibility of speeding up simulations for realistic models.

When the modulational amplitude reaches a certain limit, the extrapolation at infinity becomes negative, and, therefore, according to this scenario the entire phase space is unstable. This is in agreement with experiments $[10,11]$ that for large modulations show that the beam has a finite lifetime. In these cases the exponent $\kappa$ may become negative; a decaying of the dynamic aperture approximately proportional to the logarithm of the number of turns (i.e., $\kappa=-1$ ) was observed several years ago in the superconducting supercollider (SSC; see Ref. [34]) simulations, even though it has never been published [35].

The three parameters of Eq. (2) can be evaluated using a limited set of data, and then the formula can be extrapolated to obtain the dynamic aperture at larger times: for the modulated 4D Hénon map we show that, using the data up to $10^{5}$ turns, the extrapolation to $10^{7}$ turns agrees well with tracking (within 5\%). The interpolation involves a nonlinear fit with three parameters, and some care is needed to determine the confidence level for the best fit and the error associated with extrapolations. For the LHC case, even though a direct comparison with $10^{7}$ turns is not possible, we show that extrapolation from $10^{5}$ to $10^{6}$ turns is in agreement with tracking, and the errors associated with the extrapolation of tracking data from $10^{5}$ to $10^{7}$ turns are of the order of $5-10 \%$.

The plan of the paper is the following: as a first step, we introduce the models used for the numerical simulations, namely, the modulated Hénon map and the LHC lattice version 4.3 with tune modulation. Then we define the dynamic aperture for $N$ turns and the associated error due to the scan of the initial conditions. After that, we discuss our methods to predict a dynamic aperture. Finally, we show our numerical results for the Hénon map and the LHC, and we discuss our conclusions. 


\section{MODELS}

The study of single-particle dynamics in the presence of nonlinear forces requires an appropriate lattice model for computer simulations. The model must be, at the same time, simple for a fast computational response, and realistic enough to provide useful information. In our study we will use two models, with complementary characteristics. The 4D Hénon map [5] contains most of the physical features of a nonlinear lattice, in particular a 4D description of the motion with the possibility to include tune modulation. Its implementation is straightforward, and provides a very fast computer tracking simulation, well suited to investigate longterm dynamical behaviors in detail. On the other hand, the LHC lattice model allows one to investigate $6 \mathrm{D}$ symplectic motion, and provides quantitative information on the effects of a realistic set of multipolar errors.

The tune ripple has a strong destabilizing effect on nonlinear motion. In order to investigate scaling laws, we decided, somewhat arbitrarily, to introduce in our simulations a tune modulation similar to that observed in the CERN-SPS in various occasions. In the SPS, the instantaneous tunes can be continuously measured with a Schottky noise detector [36] at constant energy, in steady-state conditions. Peak-topeak variations of the order of $2-3$ in $10^{-4}$ units are usually observed. The frequency spectrum is mostly made of seven dominant peaks: the main one at $50 \mathrm{~Hz}$ and the others at the higher harmonics of $50 \mathrm{~Hz}$.

In our computer simulations, the time variation of the tune is represented by the sum of seven sine waves, with the same frequencies and amplitudes observed in the SPS spectrum. The modulation amplitude can be changed globally by a multiplicative factor applied to each component.

\section{A. Modulated Hénon map}

The modulated 4D Hénon map reads

$$
\left(\begin{array}{c}
x^{(n+1)} \\
p_{x}^{(n+1)} \\
y^{(n+1)} \\
p_{y}^{(n+1)}
\end{array}\right)=\mathbf{L}\left(\begin{array}{c}
x^{(n)} \\
p_{x}^{(n)}+\left[x^{(n)}\right]^{2}-\left[y^{(n)}\right]^{2} \\
y^{(n)} \\
p_{y}^{(n)}-2 x^{(n)} y^{(n)}
\end{array}\right),
$$

where $\left(x, p_{x}, y, p_{y}\right)$ are the phase space coordinates, and the linear part of the map $\mathbf{L}$ is the direct product of two twodimensional rotations $R$,

$$
\mathbf{L}=\left(\begin{array}{cc}
R\left(\omega_{x}^{(n)}\right) & 0 \\
0 & R\left(\omega_{y}^{(n)}\right)
\end{array}\right),
$$

whose linear frequencies $\omega_{x}^{(n)}, \omega_{y}^{(n)}$ are slowly varying with the discrete time $n$ according to

$$
\begin{aligned}
& \omega_{x}^{(n)}=\omega_{x 0}\left(1+\epsilon \sum_{k=1}^{m} \epsilon_{k} \cos \left(\Omega_{k} n\right)\right), \\
& \omega_{y}^{(n)}=\omega_{y 0}\left(1+\epsilon \sum_{k=1}^{m} \epsilon_{k} \cos \left(\Omega_{k} n\right)\right) .
\end{aligned}
$$

We considered the parameters given in Table I: one main
TABLE I. Parameters of the modulated Hénon map.

\begin{tabular}{ccc}
\hline \hline$k$ & $\Omega_{k}$ & $10^{4} \epsilon_{k}$ \\
\hline 1 & $2 \pi / 868.12$ & 1.000 \\
2 & $2 \Omega_{1}$ & 0.218 \\
3 & $3 \Omega_{1}$ & 0.708 \\
4 & $6 \Omega_{1}$ & 0.254 \\
5 & $7 \Omega_{1}$ & 0.100 \\
6 & $10 \Omega_{1}$ & 0.078 \\
7 & $12 \Omega_{1}$ & 0.218 \\
\hline \hline
\end{tabular}

frequency $\Omega_{1}$ and six harmonics with relative amplitudes ranging from 0.7 to 0.07 . These data correspond to the tune modulation due to the observed ripple in the quadrupoles of the SPS [10]. In our simulations we fix the linear frequencies $\omega_{x 0}$ and $\omega_{y 0}$ to 0.168 and 0.201 , i.e., rather close to resonances of order 6 and 5, in order to have relevant long-term phenomena. A Hènon map without modulation with the same linear frequencies was extensively analyzed in Ref. [9]. The modulational frequencies $\Omega_{k}$ and the amplitude ratios $\epsilon_{k}$ are fixed according to the values of Table I, and we analyze the dependence of the dynamic aperture on the amplitude $\epsilon$ of the modulation, that has been varied between 1 and 64 .

\section{B. LHC with tune modulation}

The lattice of the LHC is described in Ref. [37]. It is made of 23 regular cells per arc, each containing six tightly packed 14.2-m-long dipoles. There are eight octant insertions, four experimental insertions, and four machine insertions. The experimental insertions are tuned with injection optics $\left(\beta^{*}\right.$ $=6 \mathrm{~m})$. The horizontal and the vertical tunes are slightly separated: $Q_{x}=63.28$ and $Q_{y}=63.31$. This choice results from the optimization of the LHC working point, as described in Ref. [38]. The machine superperiodicity is 1 .

The field-shape errors are described by thin-lens multipoles up to order 11, located in the middle of each dipole and quadrupole. For every magnet, each multipolar component is determined using a random number generator with Gaussian distribution, truncated at three rms deviations. The mean value of the Gaussian distribution is specific of the machine octant. This feature is typical of the LHC: the magnets are supposed to have systematic errors that vary from octant to octant. The error values of the main dipoles and quadrupoles used in the simulation are given in Ref. [39]. The selected realization of the random imperfections used in Sec. V B has a dynamic aperture at $10^{5}$ turns close to the average value in a set of 64 random realizations.

A set of multipolar elements is used to correct the nonlinear imperfections of the LHC lattice. At each dipole end there are sextupolar and decapolar correctors interconnected in families. They are intended to compensate for the average value of the sextupolar and decapolar systematic errors of each octant. Two families of sextupoles, located close to the focusing and defocusing quadrupoles, are used to correct the chromaticities. However, to partially take into account the operational difficulty of this correction in a real machine, we decided, somewhat arbitrarily, to set $Q^{\prime}=2$. Since we are interested in scaling laws for nonlinear phenomena, we decided to disregard linear imperfections that induce finite 
closed orbits or linear coupling.

The tune modulation is obtained by summing up seven sine waves with the same relative amplitudes and frequencies as those used for the Hénon map (see Table I). The global amplitude is varied by a multiplicative factor $\epsilon$ that ranges from 1 to 8 . The horizontal and vertical tunes are affected by a synchrotron modulation of the same order of magnitude as the one induced by ripple. The numerical results quoted in Sec. V B refer to particles tracked with an initial momentum deviation of $\delta p / p=10^{-4}$, resulting in a tune oscillation of $2 \times 10^{-4}$ amplitude due to synchrotron coupling.

\section{DYNAMIC APERTURE EVALUATION}

\section{A. Dynamic aperture definition}

In a previous work [27], we proposed a definition of a dynamic aperture as a function of the number of turns $N$ as the first amplitude where particle loss occurs before $N$ turns, averaged over the phase space. Particles are started along a 2D polar grid in the coordinate space $(x, y)$ :

$$
x=r \cos \theta, \quad y=r \sin \theta,
$$

and the initial momenta $p_{x} p_{y}$ are set to zero. Let $r(\theta ; N)$ be the last stable initial condition along $\theta$ before the first loss at a turn number lower than $N$ occurs. Then the dynamic aperture is defined as

$$
D=\left(\int_{0}^{\pi / 2}[r(\theta ; N)]^{4} \sin 2 \theta d \theta\right)^{1 / 4}
$$

With respect to the approach used in several long-term simulations (see, for instance, Refs. [6,26,38]), where a fixed value of $\theta$ is considered in order to speed up simulations, this definition provides a smoother dependence of $D$ on $N$, thus allowing one to derive interpolating formulas and to extrapolate them to predict long-term particle loss.

\section{B. Error estimate}

One of the crucial issues in the definition of the dynamic aperture is the determination of the associated error. When definition (7) is implemented in a computer code, one has to carry out two discretizations: one over the radial variable $r$ and one over the angular variable $\theta$. Let $\Delta r=\left(r_{\max }\right.$ $\left.-r_{\min }\right) / N_{r}$ and $\Delta \theta=\pi /\left(2 N_{\theta}\right)$ be the step sizes in $r$ and $\theta$, respectively. The total error can be obtained using a Gaussian sum in quadrature

$$
\Delta D=\sqrt{\left(\frac{\partial D}{\partial r} \frac{\Delta r}{2}\right)^{2}+\left(\frac{\partial D}{\partial \theta} \frac{\Delta \theta}{2}\right)^{2}}
$$

An approximated formula for the error can be obtained by replacing the dynamic aperture definition with a simple average over $\theta$,

$$
D=\frac{2}{\pi} \int_{0}^{\pi / 2} r(\theta ; N) d \theta \equiv\langle r(\theta ; N)\rangle .
$$

Using this formula, the associated error reads

$$
\Delta D=\sqrt{\frac{(\Delta r)^{2}}{4}+\left\langle\left|\frac{\partial r}{\partial \theta}\right|\right\rangle^{2} \frac{(\Delta \theta)^{2}}{4}},
$$

and therefore it turns out that the step in $r$ must be equal to the step in $\theta$ times $\langle|\partial r / \partial \theta|\rangle$ to optimize the integration steps.

\section{DYNAMIC APERTURE PREDICTION}

\section{A. Dynamic aperture extrapolation}

\section{Interpolating law}

In previous works $[9,32]$, we showed that survival plots can be interpolated by a simple formula provided that the definition of dynamic aperture (7) is used. In fact, the dynamic aperture turns out to decay with the inverse of the logarithm of the number of turns, and to have a limit $A$ for infinite number of turns that in general is positive,

$$
D(N)=A+\frac{B}{\log _{10}(N)} .
$$

It has been pointed out [28] that Eq. (11) can be justified in terms of the Kolmogorov-Arnold-Moser (KAM) of the Nekhoroshev theorems, using a simple model of phase space. One assumes that the phase space is divided into two parts.

(i) An inner region where almost all the initial conditions give rise to regular orbits, except a negligible fraction of initial conditions that falls on the resonance web (the Arnold web). According to the terminology of Ref. [15], we have only thin layer diffusion. We assume that this domain can be considered stable for infinite times.

(ii) An outer region where almost all the initial conditions give rise to chaotic orbits, except a small fraction of regular orbits around stable islands, that can be neglected. According to the terminology of Ref. [15] we have thick layer diffusion, i.e., most of the invariant surfaces have been wiped out. Particles escape from this region according to the Nekhoroshev exponential estimate, since they are close to the chain of the last invariant tori that are on the border of the previous region.

Numerical simulations based on long-term tracking and frequency analysis have confirmed this scenario for 4D mappings [9]. Using these hypotheses, one can work out the following formula:

$$
D(N)=A+\frac{B}{\log _{10}^{\kappa}\left(N / N_{0}\right)} .
$$

The optimal analytical estimate of the exponent $\kappa$ is equal to $(d-1) / 2$, where $d$ is the phase space dimension [40]. For instance, in the case of a four-dimensional map (such as the Hénon map without modulation or the LHC on momentum, without modulation) one has $d=4$, and therefore $\kappa=1.5$. One can try to interpolate the long-term data using $A, B$, and $\kappa$ as free parameters, and fixing $N_{0}$ to one using the heuristic argument $D(1) \approx \infty$. It turns out (see Ref. [32]) that in several cases the fitted value of the exponent agrees with the analytical estimate. In the case with tune modulation, one can make the following observations. 
(i) From a theoretical point of view, the Nekhoroshev theorem holds for weak perturbations of an integrable Hamiltonian that is given by a certain number of uncoupled oscillators. Adding the modulation of the linear frequencies strongly modifies the structure of the Hamiltonian, and therefore Eq. (12) can no longer be justified in terms of the Nekhoroshev theorem.

(ii) From a phenomenological point of view, it is evident that when the modulation is added the long-term phenomena become more relevant. On the other hand, Eq. (12) means that the lower $\kappa$ is, the more relevant the long-term phenomena are. Therefore, if Eq. (12) is still valid with modulation, the exponent $\kappa$ should decrease with the amplitude of the modulation.

(iii) Finally, we point out that when the modulation becomes very relevant, both numerical simulations and real experiments show that all the phase space becomes unstable. This behavior can be taken into account by Eq. (12) if $\kappa$ is negative, or if $\kappa$ is positive but $A$ is negative.

Summarizing, we point out that Eq. (12) is not theoretically justified to interpolate the data in the case of tune modulation. Nevertheless, if we consider $\kappa$ as an additional free parameter to fit the data, Eq. (12) can model cases where the long-term phenomena are very relevant, up to the extreme situation where the entire phase space is unstable. For this reason we also propose to use Eq. (12) to interpolate the long-term data in the case with modulation. We will show that the numerical data are very well interpolated by Eq. (12); this implies that the model with an inner stable region and an outer chaotic region is still valid for the modulated case; the interpolation means that the effect of the modulation is to shrink the stable core and to slow down the escape rate of the initial conditions in the outer region.

\section{Errors of the fit and extrapolation}

The fitting procedure was carried out using the standard approach based on least-squares minimization. Assuming that the evaluation of the dynamic aperture is affected by Gaussian-distributed errors, the function

$$
\chi^{2}(A, B, \kappa)=\frac{1}{N-3} \sum_{i=1}^{N}\left[\frac{y_{i}-A-B / \log _{10}^{\kappa}\left(x_{i}\right)}{\sigma_{i}}\right]^{2}
$$

follows a $\chi^{2}$ distribution, and the parameters $A, B$, and $\kappa$ that minimize $\chi^{2}$ are the maximum likelihood estimators. In the previous formula, $y_{i}$ is the dynamic aperture evaluated at $x_{i}$ turns, and $\sigma_{i}$ is the associated error (see Sec. III).

For our purposes, it is important to compute not only the best value of the parameters, but also to evaluate the associated errors. Since the fit is nonlinear in $\kappa$, no analytical formula is available, either for the best parameters or for the errors. A scan over $\kappa$ is carried out: for each value of $\kappa$, the optimal values of $A$ and $B$ are worked out analytically. Among this one-parameter family of fitting values, we choose the ones that minimize the $\chi^{2}$ [see Eq. (13)]. A $\chi_{\text {min }}^{2}$ of the order of 1 ensures that the data are well fitted by the interpolating law. In order to work out the associated error, the standard technique consists in determining the set in the parameter space $(A, B, \kappa)$ that satisfy

$$
\chi^{2}(A, B, \kappa)-\chi_{\min }^{2} \leqslant \Delta \chi,
$$

where $\Delta \chi$ is related to the chosen confidence level on the parameters. We used $\Delta \chi=2.7$, that ensures a confidence level of $90 \%$ (see Ref. [42] for more details). Then, the projection of the set that satisfies Eq. (14) on the axes $A, B$, and $\kappa$ provides the confidence intervals for the best fit. In the following we show that such intervals are rather asymmetric around the best fit: this is due to the nonlinear character of the fitting function.

The extrapolation of the formula to a higher number of turns is made by using the best parameters; in order to evaluate the error, we extrapolate using all the parameters inside the deformed ellipsoid (14), and we obtain maximum and minimum extrapolation values. Also in this case the interval around the best value can be rather asymmetric.

\section{B. Lyapunov exponent}

In this section we briefly recall the definition of the maximal Lyapunov exponent $[8,9,23,21,22]$ and the method based on thresholds that was proposed in Ref. [9]; then we analyze the case with modulation. The maximal Lyapunov exponent specifies the ratio of divergence of two orbits whose initial conditions are close in phase space. The estimate of the maximal Lyapunov exponent after $N$ turns is given by

$$
\lambda(N)=\frac{1}{N} \log \frac{\left|x^{(N)}-\hat{x}^{(N)}\right|}{\delta}, \quad \delta \ll 1,
$$

where $x^{(N)}$ and $\hat{x}^{(N)}$ are the iterates of the initial conditions $x^{(0)}$ and $\hat{x}^{(0)}$, respectively, $\delta=\left|x^{(0)}-\hat{x}^{(0)}\right|$ is the initial distance, and log is the natural logarithm. The theory states that if $\lim _{N \rightarrow \infty} \lambda(N)=0$, the orbit is regular and therefore the particle is stable; if the limit is positive, the trajectory is chaotic (i.e., there is exponential divergence of nearby trajectories, and hence sensitivity to initial conditions), and therefore the particle can be lost sooner or later. The estimate of the Lyapunov coefficient with Eq. (15) allows one to determine the border between chaotic and regular motion, and therefore to predict the dynamic aperture for an infinite number of turns. However, it cannot provide quantitative information on the stability of the motion for a finite number of turns.

In Ref. [9] we proposed an automatic method to select regular from chaotic orbits based on a threshold on the Lyapunov exponent. For regular particles, the distance between neighbor orbits linearly increases with the discrete time $N$ : this is due to the dependence of the frequency on the amplitude. Therefore it is natural to fix a threshold according to

$$
\sigma_{\lambda}(N)=\frac{1}{N} \log A_{\lambda} N
$$

If $\lambda(N)>\sigma_{\lambda}(N)$, then the particle is assumed to be chaotic, while if $\lambda(N)<\sigma_{\lambda}(N)$ the particle is regular. One can show (see Ref. [9]) that the constant $A_{\lambda}$ is related to the maximum of the derivative of the tune with respect to the amplitude where the tune is well defined.

In Fig. 1 we show the distribution of the Lyapunov exponents of the initial conditions started along grid (6) for the 

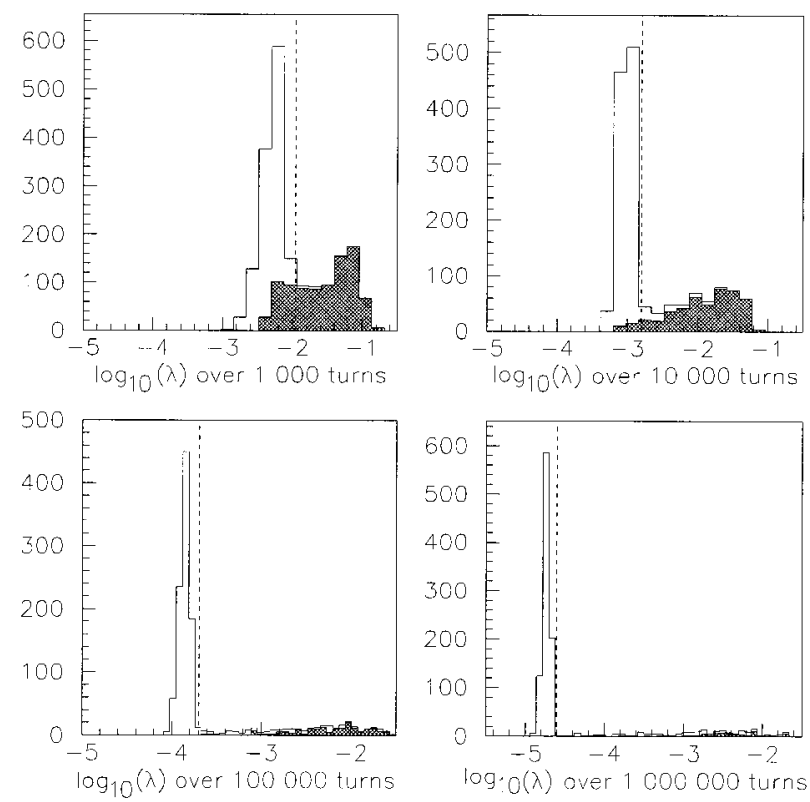

FIG. 1. Distribution of the Lyapunov exponent (in log scale) evaluated at four different numbers of turns for the Hénon map without modulation at $\omega_{x 0} / 2 \pi=0.168$ and $\omega_{y 0} / 2 \pi=0.201$; particles lost before $10^{7}$ turns are marked in black, and the dashed lines show the thresholds according to Eq. (16).

Hénon map without modulation; four different numbers of turns $N=10^{3}, 10^{4}, 10^{5}$, and $10^{6}$ have been used. The distribution of the Lyapunov is compared to the results of long-term tracking: particles stable for $10^{7}$ turns are marked in white, while the unstable ones are marked in black. Using the Lyapunov exponent, one can distinguish rather well which particles are stable and which are not stable: the sharp fall of the rather narrow peak of Fig. 1, that contains most of the stable particles, is the natural choice of the threshold $\sigma_{\lambda}(N)$ for long-term predictions. The peak becomes narrower and narrower when the number of turns is increased. It turns out that the thresholds fixed in this way (i.e., through the comparison with tracking) are very well interpolated by Eq. (16), with $A_{\lambda}=0.5$ (see Fig. 1, dotted lines). We already pointed out in Ref. [9] that the value of $A_{\lambda}$ seems to depend very weakly on the model.

The dynamic aperture prediction given by the Lyapunov exponent can be computed according to the same formula [see Eq. (7)], where now $r(\theta ; N)$ is the initial condition along $\theta$ whose Lyapunov exponent is below the threshold. The error associated with the Lyapunov prediction can be evaluated using the same scheme provided for plain tracking data.

In the case of tune modulation, the results are rather interesting. In Fig. 2 we show the same plot of Fig. 1 for the Hénon map with a modulation amplitude $\epsilon=4$. Also in this case, there is a peak of stable particles with a rather sharp fall on the right; the threshold is very well interpolated by Eq. (16) with the same value of the constant $A_{\lambda}$ (see the dashed lines in Fig. 2). Moreover, in this case the peak of stable particles also becomes narrower for a larger number of turns. The only difference is that the fraction of initial conditions whose Lyapunov exponent is above the threshold becomes larger with respect to the previous case. Most of these particles are not lost before $10^{7}$ turns, but according to our
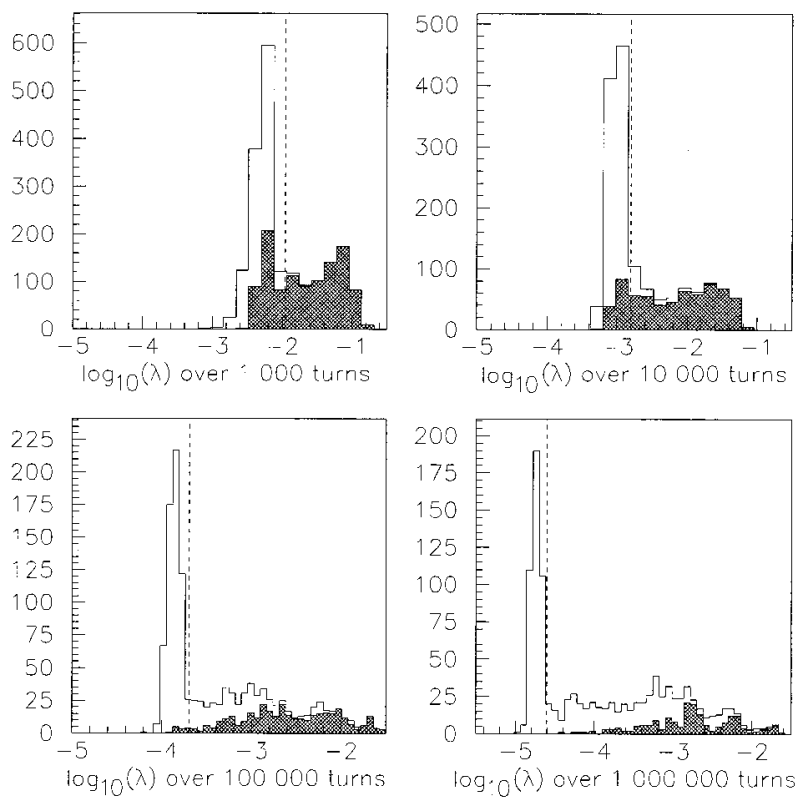

FIG. 2. Distribution of the Lyapunov exponent (in log scale) evaluated at four different numbers of turns for the modulated Hénon map with $\epsilon=4$ at $\omega_{x 0} / 2 \pi=0.168$ and $\omega_{y 0} / 2 \pi=0.201$; particles lost before $10^{7}$ turns are marked in black, and the dashed lines show the thresholds according to Eq. (16).

method they are chaotic, and therefore they may be lost at a higher number of turns.

\section{NUMERICAL RESULTS}

\section{A. Hénon map}

\section{Interpolation of tracking data}

We considered a modulated Hénon map with parameters fixed to the values specified in Sec. II A, and varied the amplitude of the modulation $\epsilon$ [see Eq. (5)] from 1 to 64. We also considered a case without modulation. Long-term tracking has been carried out up to $10^{7}$ turns, using a scan over 30 angles and 100 radial steps to optimize the error, which is around $2 \%$. In Table II we give the dynamic aperture and the associated error [see Eq. (10)] versus the number of turns for different values of $\epsilon$. As expected, the modulation has no effect over the short-term dynamic aperture $\left(10^{3}\right.$ turns $)$, while it leads to a stability loss that increases with the number of turns and with the amplitude of the modulation.

We interpolated the dynamic aperture versus the number of turns according to Eq. (12). The value of $\chi^{2}$, and of the parameters $\kappa, A$, and $B$, with the error estimated with a con-

TABLE II. Dynamic aperture (arbitrary units) vs number of turns for the modulated Hénon map.

\begin{tabular}{lccc}
\hline \hline$\epsilon$ & $\mathcal{D}\left(10^{3}\right)$ & $\mathcal{D}\left(10^{5}\right)$ & $\mathcal{D}\left(10^{7}\right)$ \\
\hline 0 & $0.57 \pm 0.01$ & $0.49 \pm 0.01$ & $0.47 \pm 0.01$ \\
1 & $0.57 \pm 0.01$ & $0.49 \pm 0.01$ & $0.46 \pm 0.01$ \\
4 & $0.57 \pm 0.01$ & $0.49 \pm 0.01$ & $0.44 \pm 0.01$ \\
16 & $0.57 \pm 0.01$ & $0.47 \pm 0.01$ & $0.40 \pm 0.01$ \\
64 & $0.57 \pm 0.01$ & $0.45 \pm 0.01$ & $0.33 \pm 0.01$ \\
\hline \hline
\end{tabular}


TABLE III. Fitting parameters of Eq. (12) for the modulated Hénon map.

\begin{tabular}{lcccc}
\hline \hline$\epsilon$ & $\chi^{2}$ & $\kappa$ & $A$ & $B$ \\
\hline 0 & 1.5 & $1.4_{-0.5}^{+0.5}$ & $0.43_{-0.06}^{+0.03}$ & $0.6_{-0.1}^{+0.3}$ \\
1 & 1.7 & $1.2_{-0.5}^{+0.5}$ & $0.40_{-0.09}^{+0.04}$ & $0.6_{-0.1}^{+0.2}$ \\
4 & 2.3 & $0.6_{-0.4}^{+0.5}$ & $0.24_{-0.56}^{+0.13}$ & $0.6_{-0.0}^{+0.5}$ \\
16 & 0.9 & $0.1_{-0.5}^{+0.4}$ & -1.5 & 2.3 \\
64 & 3.7 & $-0.5_{-0.3}^{+0.4}$ & $1.0_{-0.2}^{+2.0}$ & $-0.3_{-2.0}^{+0.2}$ \\
\hline \hline
\end{tabular}

fidence level of $90 \%$, are given in Table III. The dynamic aperture estimate through tracking with the associated error, the best fit through Eq. (12) (solid line), and the extrapolation to infinity (dotted line) are shown in Figs. 3-7. We also plotted the dynamic aperture estimate provided by the Lyapunov exponent (stars). The main results of this analysis are the following.

(i) Goodness of the fit. In all cases, the fit is extremely good ( $\chi^{2}$ is of the order of 1$)$. This is somewhat unexpected, since we applied an interpolating law outside its expected validity limits.

(ii) Parameter dependence on $\epsilon$. Both $\kappa$ and $A$ decrease as the modulational amplitude $\epsilon$ becomes larger, as expected. For small $\epsilon, B$ seems to be independent of the amplitude. For $\epsilon=16, A$ becomes negative and, therefore, according to the extrapolation, all initial conditions will be lost sooner or later.

(iii) Errors of the fit. The errors associated with the fitting parameters are rather large. In particular, the exponent $\kappa$ is determined within 0.5 for all the cases. The errors on $A$ and $B$ become larger when the modulation is increased: $A$, which

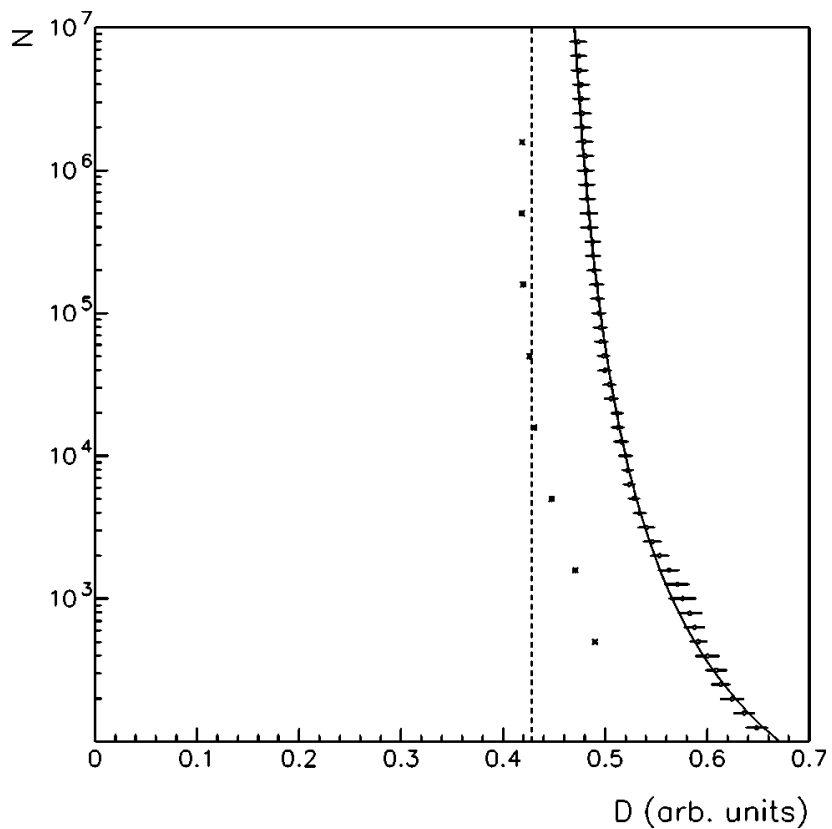

FIG. 3. Dynamic aperture $D$ vs number of turns $N$ for the Hénon map without modulation $(\epsilon=0)$. Tracking data (error bars), interpolation according to Eq. (12) (solid line) and extrapolation at infinity (vertical dotted line), and prediction through the Lyapunov exponent (stars).

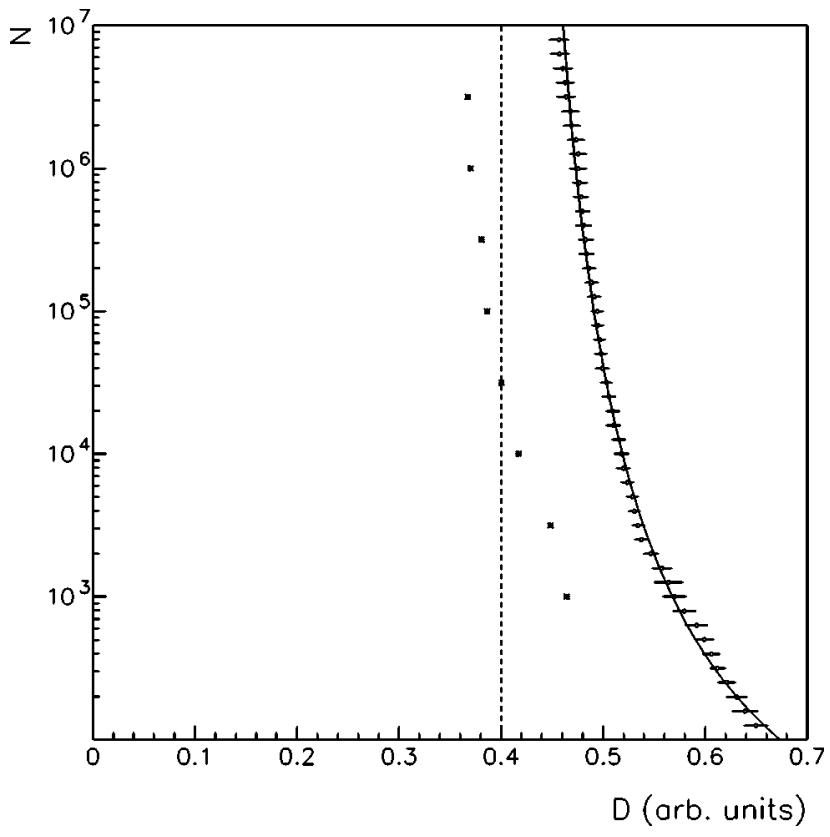

FIG. 4. Dynamic aperture $D$ vs number of turns $N$ for the modulated Hénon map $(\epsilon=1)$. Tracking data (error bars), interpolation according to Eq. (12) (solid line) and extrapolation at infinity (vertical dotted line), and prediction through the Lyapunov exponent (stars).

denotes the extrapolation of the dynamic aperture for infinite number of turns when $\kappa$ is positive, is rather sharply defined for $\epsilon=0$, but becomes rather loose when $\epsilon$ is increased. When $\kappa$ changes sign in the interval of $90 \%$ confidence level (i.e., $\epsilon=16$ ), it becomes impossible to associate an error to $A$ and $B$, since our formula contains a singularity for $\kappa=0$.

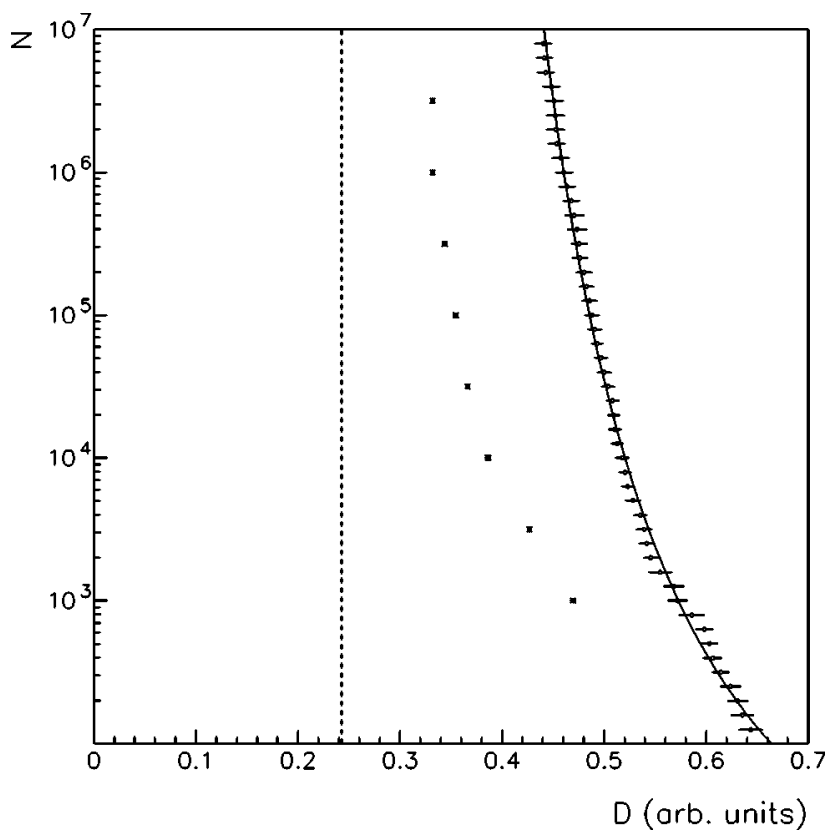

FIG. 5. Dynamic aperture $D$ vs number of turns $N$ for the modulated Hénon map $(\epsilon=4)$. Tracking data (error bars), interpolation according to Eq. (12) (solid line) and extrapolation at infinity (vertical dotted line), and prediction through the Lyapunov exponent (stars). 


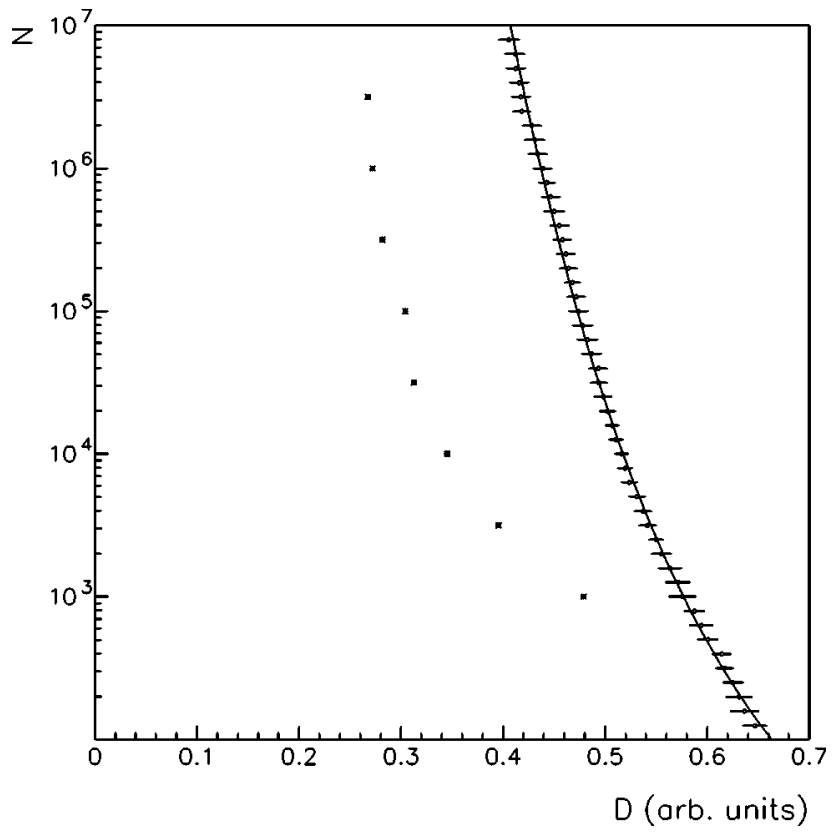

FIG. 6. Dynamic aperture $D$ vs number of turns $N$ for the modulated Hénon map $(\epsilon=16)$. Tracking data (error bars), interpolation according to Eq. (12) (solid line), and prediction through the Lyapunov exponent (stars).

We also tried a weighted fit in order to improve the precision in the determination of the parameters $A, B$, and $\kappa$; we tried different weights, without finding any significant improvement.

\section{Extrapolation}

Formula (12) allows one to extrapolate the dynamic aperture at a given number of turns using a limited set of long-

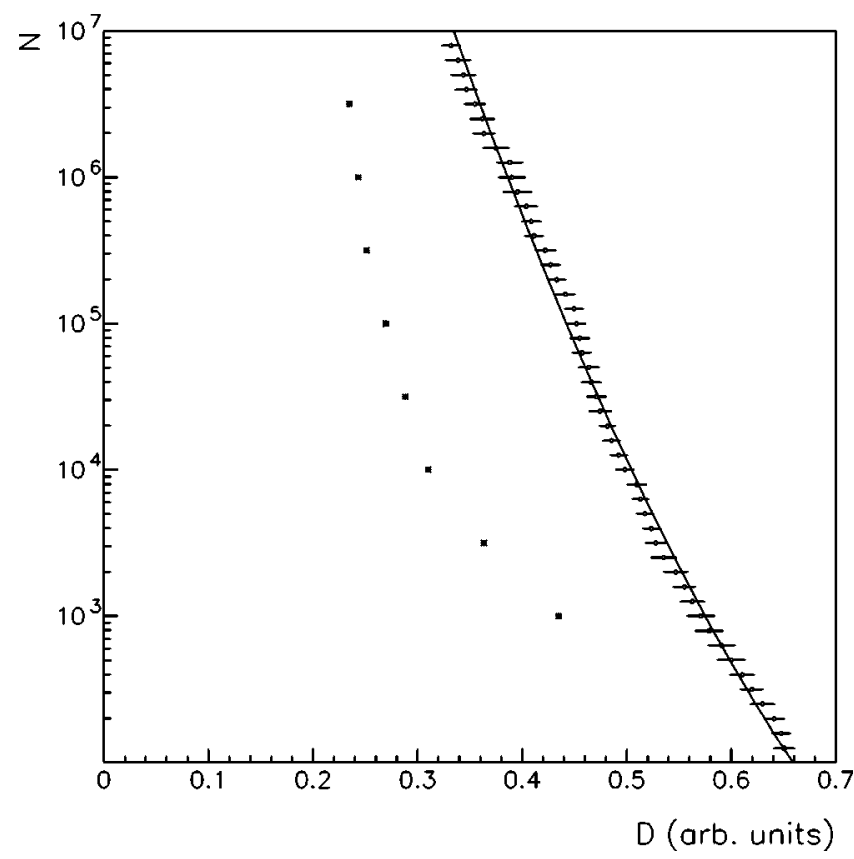

FIG. 7. Dynamic aperture $D$ vs number of turns $N$ for the modulated Hénon map ( $\epsilon=64)$. Tracking data (error bars), interpolation according to Eq. (12) (solid line), and prediction through the Lyapunov exponent (stars).
TABLE IV. Comparison between extrapolation of dynamic aperture at $10^{7}$ and tracking for the modulated Hènon map.

\begin{tabular}{|c|c|c|c|c|}
\hline \multirow[t]{2}{*}{$\epsilon$} & \multicolumn{3}{|c|}{ Extrapolation to $10^{7}$ from } & \multirow{2}{*}{$\begin{array}{l}\text { Tracking } \\
\text { (at } 10^{7} \text { ) }\end{array}$} \\
\hline & $10^{4}$ & $10^{5}$ & $10^{6}$ & \\
\hline 0 & $0.42_{-0.12}^{+0.06}$ & $0.46_{-0.03}^{+0.02}$ & $0.47_{-0.02}^{+0.02}$ & $0.47_{-0.01}^{+0.01}$ \\
\hline 1 & $0.41_{-0.10}^{+0.05}$ & $0.46_{-0.02}^{+0.02}$ & $0.46_{-0.01}^{+0.01}$ & $0.46_{-0.01}^{+0.01}$ \\
\hline 4 & $0.40_{-0.11}^{+0.06}$ & $0.45_{-0.03}^{+0.02}$ & $0.45_{-0.02}^{+0.01}$ & $0.44_{-0.01}^{+0.01}$ \\
\hline 16 & $0.36_{-0.17}^{+0.09}$ & $0.41_{-0.05}^{+0.03}$ & $0.41_{-0.01}^{+0.02}$ & $0.40_{-0.01}^{+0.01}$ \\
\hline 64 & $0.33_{-0.17}^{+0.10}$ & $0.37_{-0.04}^{+0.03}$ & $0.36_{-0.02}^{+0.02}$ & $0.33_{-0.01}^{+0.01}$ \\
\hline
\end{tabular}

term data. The intrinsic limits of this method lie in the error associated to the determination of the parameters $A, B$, and $\kappa$. We use tracking data from $10^{2}$ up to $N$, with $N$ $=10^{4}, 10^{5}$, and $10^{6}$, to evaluate the three parameters of Eq. (12), and then we extrapolate at $10^{7}$. We choose this set of parameters to be consistent with the simulations in the LHC. The results (see Table IV) are good: all the extrapolations are in agreement with direct tracking, even though interpolation from $10^{2}$ to $10^{4}$ provides a rather large error when extrapolated at $10^{7}$. Interpolation from $10^{2}$ to $10^{5}$ is rather precise (within 5\%); this already allows one to save a factor 100 in simulations.

In comparison with the results of Ref. [9], we note that a larger number of turns is required to obtain a reliable optimization. This is due to the fact that the effect of the modulation on the beam stability requires a longer time to become evident.

\section{Comparison with the Lyapunov exponent}

For the case without modulation the extrapolation of the dynamic aperture at infinity $A$ has a rather small error, and can be compared to the prediction of the limit of regular motion as given by the Lyapunov exponent. The agreement is good (see Fig. 3), thus supporting previous results discussed in Ref. [32]. When a small tune modulation is considered (see Figs. 4 and 5), the Lyapunov prediction converges to a rather well-defined limit that fits into the confidence interval associated to $A$. Indeed, since the error associated with $A$ is very large (see Table III), the quantitative agreement is rather loose. For large modulation amplitudes ( $\epsilon=16$ and 64; see Figs. 6 and 7), according to the extrapolation the entire phase space is unstable. On the other hand, it is hard to say whether the Lyapunov exponent predicts a finite stability domain or not.

Summarizing, in the case without modulation the border of the chaotic region evaluated through the Lyapunov exponent is in agreement with the extrapolation of our fit to infinity. When the modulation is switched on, the agreement between these two quantities becomes worse. Under these conditions it is not clear whether the stability border predicted with the Lyapunov exponent tends to a well-defined limit. Therefore, it seems very hard to extract quantitative information on the long-term stability from the Lyapunov exponent in the case with tune modulation.

\section{B. LHC}

The dynamic aperture is given in millimeters normalized at $\beta_{\max }=182 \mathrm{~m}$. Very onerous simulations have been carried 
TABLE V. Dynamic aperture $(\mathrm{mm})$ vs number of turns for the LHC.

\begin{tabular}{cccccc}
\hline \hline$\epsilon$ & $\Delta p / p$ & $\mathcal{D}\left(10^{3}\right)$ & $\mathcal{D}\left(10^{4}\right)$ & $\mathcal{D}\left(10^{5}\right)$ & $\mathcal{D}\left(10^{6}\right)$ \\
\hline 0 & 0 & $13.1 \pm 0.3$ & $12.6 \pm 0.2$ & $12.4 \pm 0.2$ & $12.3 \pm 0.2$ \\
0 & $10^{-4}$ & $12.9 \pm 0.2$ & $12.3 \pm 0.2$ & $11.8 \pm 0.2$ & $11.5 \pm 0.2$ \\
1 & $10^{-4}$ & $12.9 \pm 0.2$ & $12.2 \pm 0.2$ & $11.7 \pm 0.2$ & $11.1 \pm 0.2$ \\
2 & $10^{-4}$ & $12.9 \pm 0.2$ & $12.1 \pm 0.2$ & $11.5 \pm 0.2$ & $10.7 \pm 0.2$ \\
4 & $10^{-4}$ & $12.8 \pm 0.2$ & $11.9 \pm 0.2$ & $11.1 \pm 0.2$ & $10.4 \pm 0.2$ \\
8 & $10^{-4}$ & $13.0 \pm 0.2$ & $11.8 \pm 0.2$ & $10.8 \pm 0.2$ & $10.1 \pm 0.2$ \\
\hline \hline
\end{tabular}

out up to $10^{6}$ turns, with a scan over 17 angles and 100 radii. The relative error in the dynamic aperture is of the order of $2 \%$. In Table $\mathrm{V}$ we give the dynamic aperture as a function of $\epsilon$ and $N$. Also in this case, the effect of modulation at $10^{3}$ turns is very small (less than $2 \%$ ), while at $10^{6}$ turns it becomes relevant (around 20\%). We have carried out simulations for a beam on momentum and without modulation: this is a purely 4D model. Then we considered a beam with some off-momentum $\left(10^{-4}\right)$, and switched the modulation from $\epsilon=0$ to $\epsilon=8$.

The interpolation (see Table VI and Figs. 8-13) is very good. The best fit has a $\chi_{\min }^{2}$ that ranges from 0.4 to 2.0. The exponent decreases with the amplitude as in the Hènon case, even though the error is larger.

The extrapolation has been carried out from $10^{2}-10^{4}$ and $10^{2}-10^{5}$ up to $10^{6}$ turns. All the extrapolations are in agreement with tracking at $10^{6}$ turns, and the error for the extrapolation of $10^{2}-10^{5}$ to $10^{6}$ turns is less than $5 \%$ (see Table VII). We also tried an extrapolation to $10^{7}$ turns, even though in this case we do not have tracking data to compare with (see Table VIII). All the estimates are compatible, and using data up to $10^{5}$ turns the extrapolation up to $10^{7}$ turns has an error of the order of $5-10 \%$.

The comparison with the Lyapunov prediction is also rather difficult in this case (see Figs. 8-13). For the purely 4D case the Lyapunov seems to converge to the extrapolated value of the dynamic aperture. When the modulation is switched on, it is not evident if the Lyapunov prediction converges as before; the last two cases (see Figs. 12 and 13) clearly show that the Lyapunov exponent "feels" the instability due to the increased modulation, even though the quantitative information does not seem to be significant.

Summarizing, the LHC data show the same features of the modulated Hènon map: good interpolation, rather large errors on the fitting parameters, rather precise extrapolation for one-two orders of magnitude, and quantitative agreement of

TABLE VI. Fitting parameters of Eq. (12) for the LHC.

\begin{tabular}{cccccc}
\hline \hline$\epsilon$ & $\Delta p / p$ & $\chi^{2}$ & \multicolumn{1}{c}{$\kappa$} & \multicolumn{1}{c}{$A$} & \multicolumn{1}{c}{$B$} \\
\hline 0 & 0 & 0.4 & $1.9_{-1.2}^{+1.1}$ & $12.0_{-1.7}^{+0.3}$ & $9_{-3}^{+9}$ \\
0 & $10^{-4}$ & 1.0 & $0.8_{-1.1}^{+1.0}$ & 9.6 & 8 \\
1 & $10^{-4}$ & 1.4 & $0.3_{-1.9}^{+0.0}$ & 3.4 & 13 \\
2 & $10^{-4}$ & 2.0 & $-0.1_{-0.8}^{+0.9}$ & 42 & -26 \\
4 & $10^{-4}$ & 1.0 & $-0.1_{-0.7}^{+0.7}$ & 47 & -30 \\
8 & $10^{-4}$ & 1.3 & $-0.2_{-0.5}^{+0.5}$ & 33 & -16 \\
\hline \hline
\end{tabular}

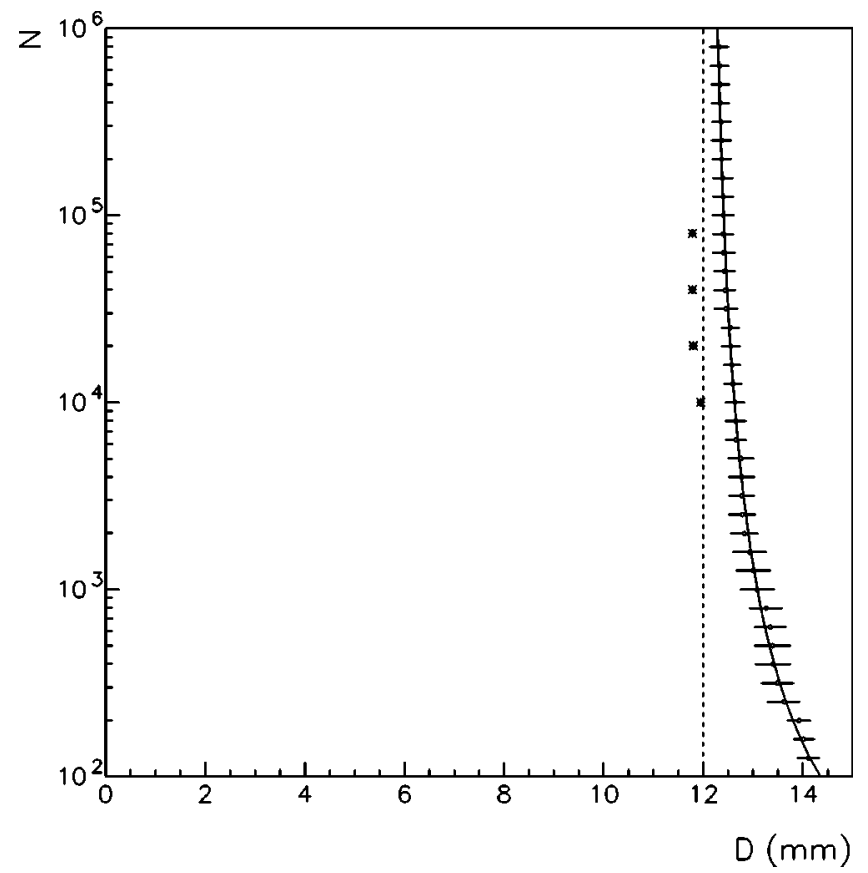

FIG. 8. Dynamic aperture $D$ vs number of turns $N$ for the LHC on momentum, without modulation $(\epsilon=0)$. Tracking data (error bars), interpolation according to Eq. (12) (solid line) and extrapolation at infinity (vertical dotted line), and prediction through the Lyapunov exponent (stars).

the Lyapunov prediction with the extrapolation of our formula only in the case without modulation.

\section{CONCLUSIONS}

In this paper we proposed an empirical formula to interpolate the dynamic aperture versus the number of turns in

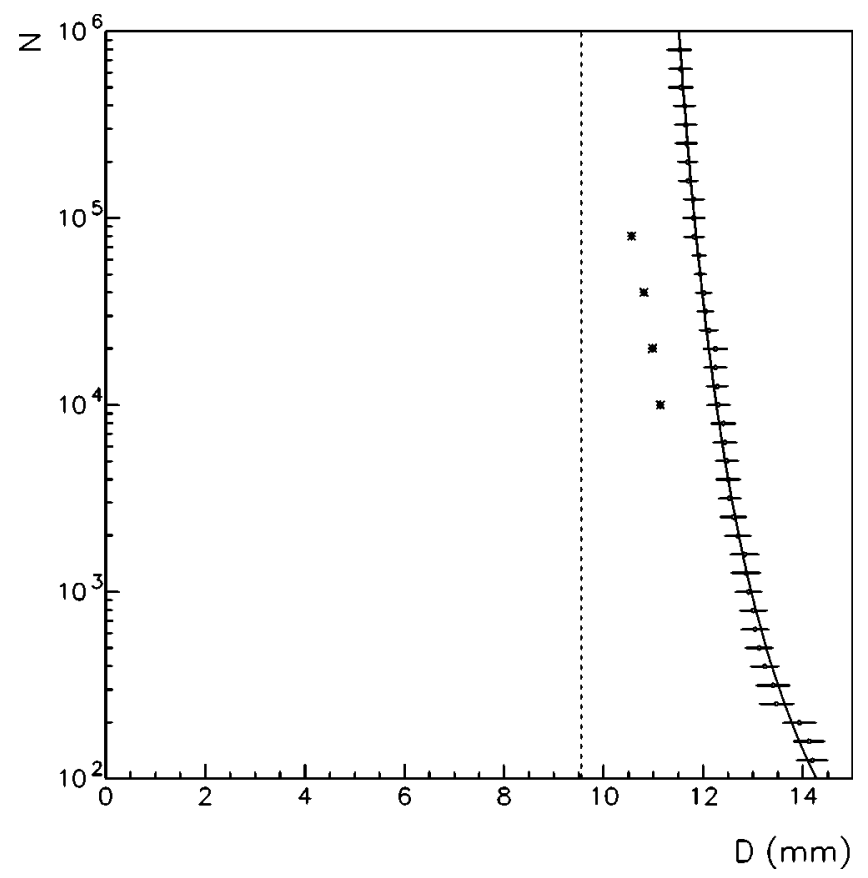

FIG. 9. Dynamic aperture $D$ vs number of turns $N$ for the LHC off momentum $\left(\Delta p / p=10^{-4}\right)$, without modulation $(\epsilon=0)$. Tracking data (error bars), interpolation according to Eq. (12) (solid line) and extrapolation at infinity (vertical dotted line), and prediction through the Lyapunov exponent (stars). 


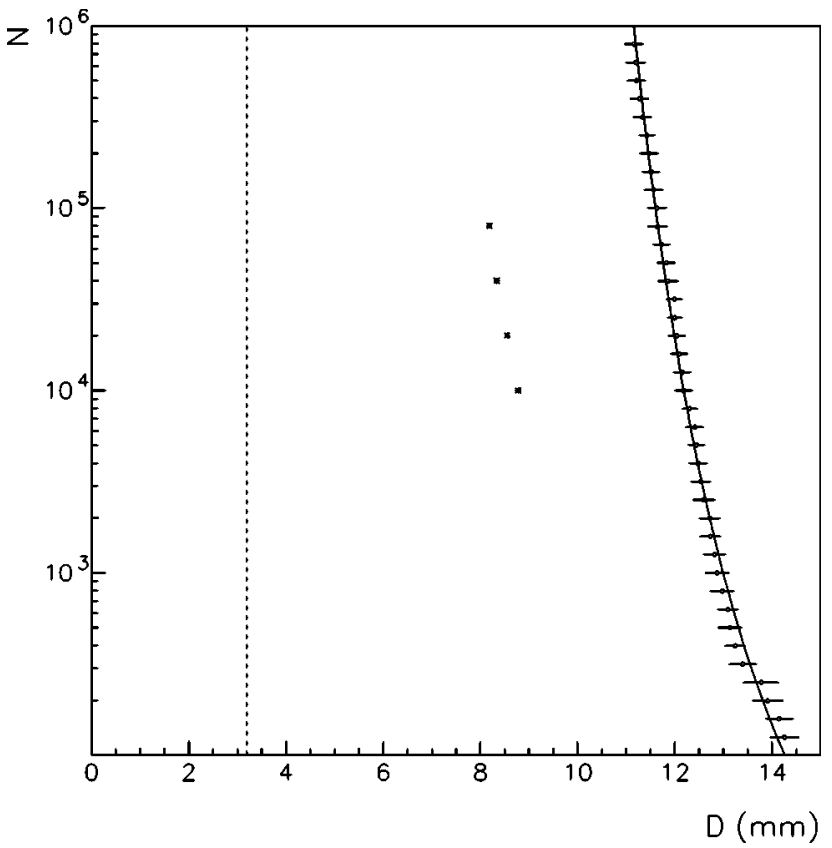

FIG. 10. Dynamic aperture $D$ vs number of turns $N$ for the LHC off momentum $\left(\Delta p / p=10^{-4}\right)$, with modulation $(\epsilon=1)$. Tracking data (error bars), interpolation according to Eq. (12) (solid line) and extrapolation at infinity (vertical dotted line), and prediction through the Lyapunov exponent (stars).

presence of tune modulation. This formula provides a quantitative framework to analyze the long-term dynamic aperture, which turns out to decay with a power of the inverse logarithm of the number of turns. Without tune modulation the exponent of the logarithm is around 1.5 both for the LHC model and the 4D Hénon map, in agreement with the analytical estimates based on the Nekhoroshev theorem [40].

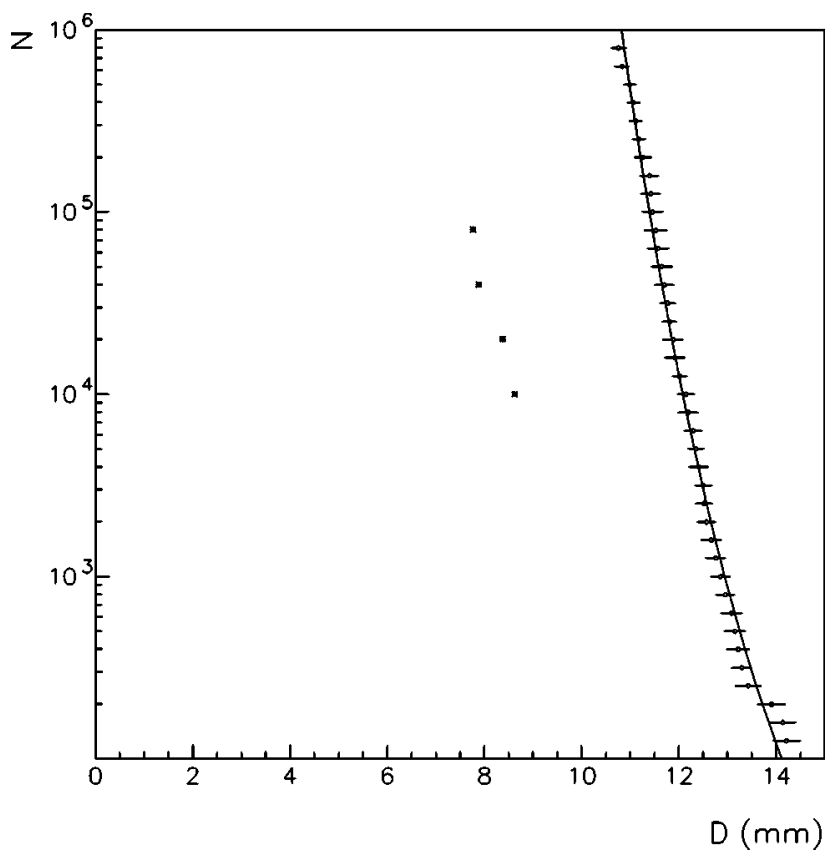

FIG. 11. Dynamic aperture $D$ vs number of turns $N$ for the LHC off momentum $\left(\Delta p / p=10^{-4}\right)$, with modulation $(\epsilon=2)$. Tracking data (error bars), interpolation according to Eq. (12) (solid line), and prediction through the Lyapunov exponent (stars).

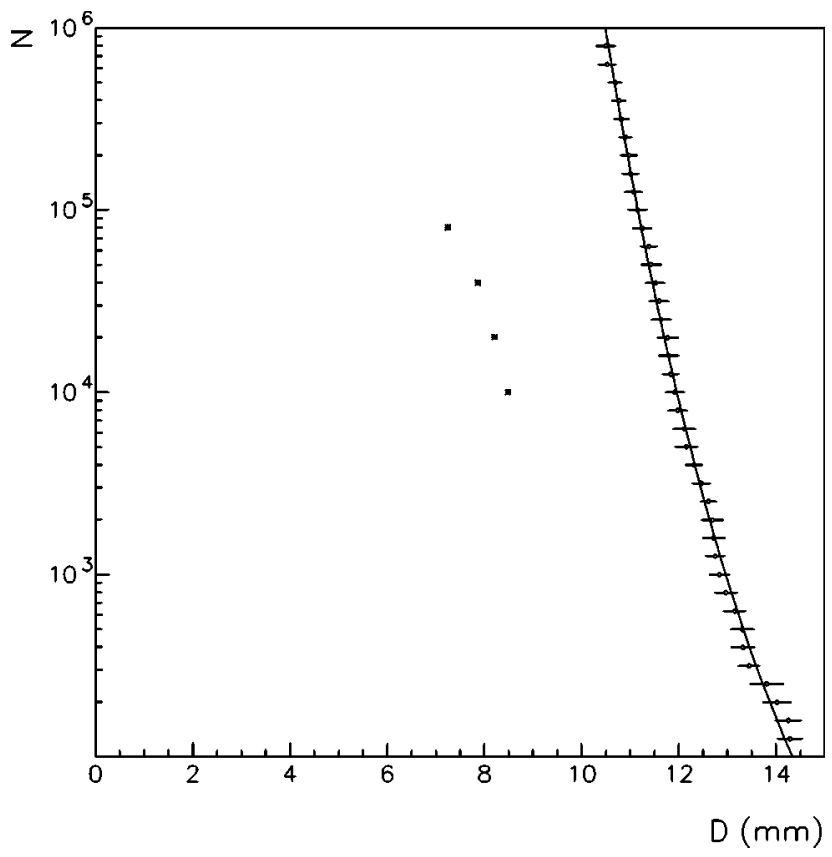

FIG. 12. Dynamic aperture $D$ vs number of turns $N$ for the LHC off momentum $\left(\Delta p / p=10^{-4}\right)$, with modulation $(\epsilon=4)$. Tracking data (error bars), interpolation according to Eq. (12) (solid line), and prediction through the Lyapunov exponent (stars).

This implies that there is a finite radius $A$, inside which the beam has an infinite lifetime. When a modulation is added, the exponent becomes larger: this makes long-term phenomena more and more relevant. It turns out that there is a monotonic dependence of the exponent on the modulational amplitude $\epsilon$. When a certain amplitude $\epsilon$ is reached, $A$ becomes negative, and therefore all the particles become unstable for

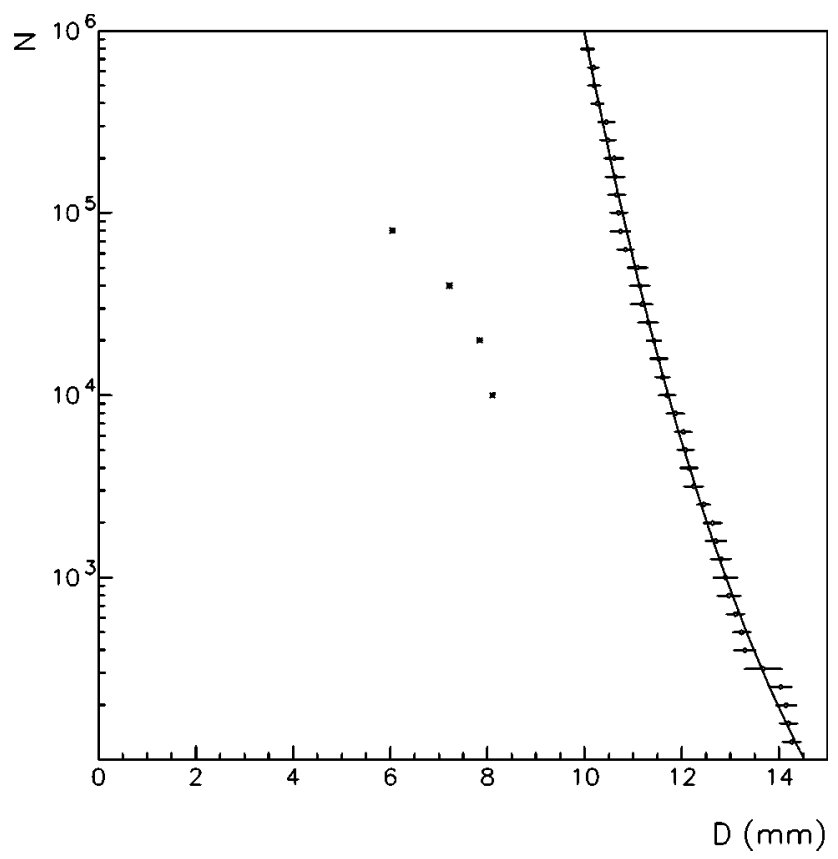

FIG. 13. Dynamic aperture $D$ vs number of turns $N$ for the LHC off momentum $\left(\Delta p / p=10^{-4}\right)$, with modulation ( $\left.\epsilon=8\right)$. Tracking data (error bars), interpolation according to Eq. (12) (solid line), and prediction through the Lyapunov exponent (stars). 
TABLE VII. Comparison between extrapolation of dynamic aperture at $10^{6}$ and tracking for the LHC.

\begin{tabular}{|c|c|c|c|c|}
\hline \multirow[t]{2}{*}{$\epsilon$} & \multirow[t]{2}{*}{$\Delta p / p$} & \multicolumn{2}{|c|}{ Extrapolation to $10^{6}$ from } & \multirow{2}{*}{$\begin{array}{c}\text { Tracking } \\
\left(\text { at } 10^{6}\right)\end{array}$} \\
\hline & & $10^{4}$ & $10^{5}$ & \\
\hline 0 & 0 & $12.2_{-1.3}^{+0.3}$ & $12.3_{-0.4}^{+0.1}$ & $12.3_{-0.2}^{+0.2}$ \\
\hline 0 & $10^{-4}$ & $11.9_{-1.0}^{+0.3}$ & $11.7_{-0.4}^{+0.4}$ & $11.5_{-0.2}^{+0.2}$ \\
\hline 1 & $10^{-4}$ & $11.8_{-1.0}^{+0.4}$ & $11.4_{-0.4}^{+0.4}$ & $11.1_{-0.2}^{+0.2}$ \\
\hline 2 & $10^{-4}$ & $11.7_{-11}^{+0.4}$ & $11.1_{-0.5}^{+0.5}$ & $10.7_{-0.2}^{+0.2}$ \\
\hline 4 & $10^{-4}$ & $10.8_{-1.9}^{+0.9}$ & $10.6_{-0.5}^{+0.5}$ & $10.4_{-0.2}^{+0.2}$ \\
\hline 8 & $10^{-4}$ & $10.1_{-2.0}^{+1.1}$ & $10.0_{-0.7}^{+0.5}$ & $10.1_{-0.2}^{+0.2}$ \\
\hline
\end{tabular}

sufficiently large times: the beam has a finite lifetime. This scenario agrees with the experiments that have been carried out on the effect on ripple on real machines [10].

The interpolation procedure involves a three-parameter nonlinear fit; we outlined the method, based on standard statistical tools, that allows one to determine the confidence level of the fit and of the extrapolation. Even though the fit parameters $A, B$, and $\kappa$ cannot be worked out with a high precision, this approach provides quantitative tools to predict long-term stability. For a realistic LHC model, the extrapolation of the dynamic aperture at $10^{7}$ turns using tracking up to $10^{5}$ turns has a small error (around 5-10\%). We checked the agreement of our extrapolation procedure against track-
TABLE VIII. Extrapolation of dynamic aperture at $10^{7}$ for the LHC.

\begin{tabular}{ccrrr}
\hline \hline$\epsilon$ & $\Delta p / p$ & \multicolumn{3}{c}{ Extrapolation to $10^{7}$ from } \\
& & $10^{4}$ & $10^{5}$ & \multicolumn{1}{c}{$10^{6}$} \\
\hline 0 & 0 & $12.0_{-1.8}^{+0.5}$ & $12.2_{-0.6}^{+0.2}$ & $12.2_{-0.2}^{+0.2}$ \\
0 & $10^{-4}$ & $11.8_{-1.5}^{+0.5}$ & $11.4_{-0.7}^{+0.4}$ & $11.3_{-0.4}^{+0.3}$ \\
1 & $10^{-4}$ & $11.7_{-1.4}^{+0.4}$ & $11.1_{-0.8}^{+0.6}$ & $10.8_{-0.4}^{+0.3}$ \\
2 & $10^{-4}$ & $11.5_{-1.5}^{+0.5}$ & $10.8_{-0.9}^{+0.6}$ & $10.3_{-0.3}^{+0.4}$ \\
4 & $10^{-4}$ & $10.4_{-3.1}^{+1.2}$ & $10.2_{-1.0}^{+0.7}$ & $9.9_{-0.3}^{+0.4}$ \\
8 & $10^{-4}$ & $9.4_{-3.5}^{+1.6}$ & $9.2_{-1.2}^{+0.8}$ & $9.4_{-0.3}^{+0.3}$ \\
\hline \hline
\end{tabular}

ing at $10^{6}$ turns, finding a good agreement. The scenario for the LHC lattice agrees with the investigations carried out for the modulated 4D Hènon map.

\section{ACKNOWLEDGMENTS}

We wish to thank Professor Turchetti for pointing out the relationship between the inverse logarithm formula and the Nekhoroshev theorem, and for giving extremely useful indications about the dependence of the exponent on the model. We also want to thank A. Chao, F. Schmidt, Y. Yan, and B. Warnock for useful discussions. M. Böge, H. Grote, C. Iselin, and E. Macintosh provided us with help and useful suggestions for long-term computer tracking.
[1] The LHC Study Group, CERN Report No. 95-05, 1995 (unpublished).

[2] A. Schoch, CERN Report No. 57-21, 1957 (unpublished).

[3] G. Guignard, CERN Report No. 76-06, 1976 (unpublished).

[4] R. D. Ruth, Lect. Notes Phys. 247, 37 (1985).

[5] A. Bazzani, E. Todesco, G. Turchetti, and G. Servizi, CERN Report No. 94-02, 1994 (unpublished).

[6] Y. Yan, Superconducting Supercollider Report No. SSC 500 (1991).

[7] A. Chao, in Nonlinear Dynamics and Particle Acceleration, edited by Yoshi H. Ichikawa and Toshiki Tajimi, AIP Conf. Proc. No. 230 (AIP, New York, 1990), p. 203.

[8] F. Schmidt, F. Willeke, and F. Zimmermann, Part. Accel. 35, 249 (1991)

[9] M. Giovannozzi, W. Scandale, and E. Todesco, Part. Accel. 56, 195 (1997).

[10] W. Fischer, M. Giovannozzi, and F. Schmidt, Phys. Rev. E 55, 3507 (1997).

[11] D. Brandt et al., in EPAC 90, edited by P. Marin and P. Mandrillon (Edition Frontières, Gif-sur-Yvette, 1991), pp. 143840.

[12] T. Chen et al., Phys. Rev. Lett. 68, 33 (1992).

[13] Y. Wang et al., Phys. Rev. E 49, 5697 (1994).

[14] B. V. Chirikov, Phys. Rep. 52-5, 263 (1979).

[15] B. V. Chirikov, M. A. Lieberman, D. L. Shepelyansky, and F. M. Vivaldi, Physica D 14, 289 (1985).

[16] O. S. Brüning, Part. Accel. 41, 133 (1993).

[17] A. I. Neishtadt, Sov. J. Plasma Phys. 12, 568 (1986).

[18] A. Bazzani, S. Siboni, and G. Turchetti, J. Phys. A 30, 27 (1997).
[19] A. Bazzani, F. Brini, and G. Turchetti, in Nonlinear Problems in Particle Accelerators: Theory and Experiments, edited by M. Cornacchia and C. Pellegrini (AIP, New York, in press).

[20] A. Gerasimov, CERN Report No. CERN-SL (AP) 92-30 (1992) (unpublished).

[21] M. Hénon and C. Heiles, Astron. J. 69-1, 73 (1964).

[22] C. Froeschlé, Astron. Astrophys. 16, 172 (1972).

[23] G. Benettin, L. Galgani, A. Giorgilli, and J. M. Strelcyn, Meccanica 15-1, 21 (1980).

[24] R. L. Warnock and R. D. Ruth, Physica D 56, 188 (1992).

[25] R. L. Warnock, in Beam Stability and Nonlinear Dynamics, edited by Z. Parsa (AIP, New York, in press).

[26] F. Galluccio and F. Schmidt, in Third European Particle Accelerator Conference, edited by H. Henke (Edition Frontiéres, Gif-sur-Yvette, 1993), pp. 640-642.

[27] E. Todesco and M. Giovannozzi, Phys. Rev. E 53, 4067 (1996).

[28] G. Turchetti (private communication).

[29] N. N. Nekhoroshev, Russ. Math. Surv. 32, 1 (1977).

[30] G. Turchetti, in Number Theory and Physics, edited by J. M. Luck and P. Moussa (Springer-Verlag, Berlin, 1990), pp. 22334.

[31] A. Bazzani, S. Marmi, and G. Turchetti, Celest. Mech. 47, 333 (1990).

[32] M. Giovannozzi, W. Scandale, and E. Todesco, in Beam Stability and Nonlinear Dynamics (Ref. [25]).

[33] M. Böge and F. Schmidt, in Beam Stability and Nonlinear Dynamics (Ref. [25]).

[34] The SSC working group, Superconducting Supercollider Re- 
port No. SSCL-SR 1056 (1990) (unpublished).

[35] D. Ritson (private communication).

[36] T. Linnecar and W. Scandale, IEEE Trans. Nucl. Sci. 30, 2185 (1983).

[37] W. Scandale et al., in PAC 95 (IEEE, Piscataway, NJ, 1995), pp. 2844-46.

[38] Z. Guo, T. Risselada, and W. Scandale, in Advanced Beam Dynamics Workshop on Effects of Errors in Accelerators, Their Diagnosis, and Corrections, edited by A. W. Chao, AIP Conf. Proc. No. 255 (AIP, New York, 1992), pp. 50-65.
[39] LHC field quality working group (private communication).

[40] A. Bazzani and G. Turchetti, in Chaotic Dynamics: Theory and Practice, edited by T. Bountis (Plenum, New York, 1991), p. 59.

[41] A. Morbidelli and A. Giorgilli, J. Stat. Phys. 78, 1607 (1995).

[42] W. H. Press, S. A. Teukolsky, W. T. Vetterling, and B. P. Flannery, Numerical Recipes in FORTRAN: The Art of Scientific Computing, 2nd ed. (Cambridge Univ. Press, Cambridge, 1992). 